\title{
The Modal-Hamiltonian Interpretation of Quantum Mechanics as a Kind of "Atomic" Interpretation
}

\author{
Juan Sebastián Ardenghi ${ }^{1}$ and Olimpia Lombardi ${ }^{2}$ \\ ${ }^{1}$ CONICET-Instituto de Astronomía y Física del Espacio, Ciudad Universitaria, CP 1429, Buenos Aires, Argentina \\ ${ }^{2}$ CONICET-Facultad de Ciencias Exactas y Naturales, Ciudad Universitaria, CP 1429, Buenos Aires, Argentina
}

Correspondence should be addressed to Olimpia Lombardi, olimpiafilo@arnet.com.ar

Received 6 May 2011; Accepted 5 August 2011

Academic Editor: Weitao Yang

Copyright ( 12011 J. S. Ardenghi and O. Lombardi. This is an open access article distributed under the Creative Commons Attribution License, which permits unrestricted use, distribution, and reproduction in any medium, provided the original work is properly cited.

\begin{abstract}
Modal interpretations are non-collapse interpretations, where the quantum state of a system describes its possible properties rather than the properties that it actually possesses. Among them, the atomic modal interpretation (AMI) assumes the existence of a special set of disjoint systems that fixes the preferred factorization of the Hilbert space. The aim of this paper is to analyze the relationship between the AMI and our recently presented modal-hamiltonian interpretation (MHI), by showing that the MHI can be viewed as a kind of "atomic" interpretation in two different senses. On the one hand, the MHI provides a precise criterion for the preferred factorization of the Hilbert space into factors representing elemental systems. On the other hand, the MHI identifies the atomic systems that represent elemental particles on the basis of the Galilei group. Finally, we will show that the MHI also introduces a decomposition of the Hilbert space of any elemental system, which determines with precision what observables acquire definite actual values.
\end{abstract}

\section{Introduction}

The modal interpretations of quantum mechanics are interpretations where measurement plays no role in the assignment of properties to physical systems. On the contrary, quantum measurements are conceived as ordinary physical interactions and measurements outcomes as properties of measurement apparatuses. Therefore, modal interpretations are non-collapse interpretations, where the quantum state of a system describes its possible properties rather than the properties that it actually possesses. The relationship between the quantum state and the values of any observable is probabilistic. Therefore, the quantum state supplies the grounds for modal statements, that is, statements about what possibly or necessarily is the case (see [1-3]). On the basis of this general idea, several modal interpretations were presented. Among them, the atomic modal interpretation (AMI, [4]) relies on the assumption that there exists a special set of disjoint systems, which are the building blocks of all the other systems, and that set fixes a preferred factorization of the Hilbert space; the properties of a composite system supervene on the properties ascribed to its "atomic" subsystems.
The main challenge for the AMI is to provide some idea about how such preferred partition of the universe should look like.

In previous works (see [5-10]), we have contributed to the family of modal interpretations with the so-called modalHamiltonian interpretation (MHI), where the Hamiltonian of the system acquires a central relevance, besides its wellknown role in representing a constant of motion and in governing the dynamics of the system. In this interpretation, the preferred context, that is, the set of observables that acquire actual values, is defined by the Hamiltonian of the closed system. In this work, our aim is to analyze the relationship between the AMI and the MHI, by showing that the MHI can be viewed as a kind of "atomic" interpretation in two different senses. On the one hand, the MHI provides a precise criterion for the factorization of the Hilbert space of the universe into factors representing elemental systems. On the other hand, the MHI identifies the atomic systems that represent elemental particles on the basis of the Galilei group. For this purpose, in Sections 2 and 3, we will introduce the AMI and the MHI interpretations, respectively. In Section 4, 
we will stress the difference between elemental systems and atomic systems in the context of the MHI, arguing that the AMI cannot stress this difference because it does not consider the difference between the non-interacting and the interacting cases. In Section 5, we will show that the MHI also introduces a decomposition of the Hilbert space of any elemental system, which determines with precision what observables acquire definite actual values. Finally, in Section 6 we will draw our conclusions.

\section{The Atomic Modal Interpretation}

The conceptual motivation of the AMI relies on an argument that runs as follows. As it is well known, the Hilbert space of the universe $\mathscr{H}^{\text {univ }}$, like any Hilbert space, can be factorized in countless ways. Then, if one supposes that each factorization defines a set of basic or atomic subsystems, the multiple factorizability of a Hilbert space implies that there exists a multiplicity of ways of defining the building blocks of nature. Since the associated states of each quantum system can be found by means of the partial trace with respect to the rest of the universe, then a Boolean algebra could be established over each atomic quantum system. But this would lead to a contradiction because of the Kochen-Specker theorem [11].

The AMI ([4]) tries to overcome this obstacle by assuming that, in nature, there is a set of mutually disjoint atomic quantum systems $8^{j}$ that conform the building blocks of all the other quantum systems. In other words, the set of the building blocks of the universe is one and only one. From the mathematical point of view, this means that the Hilbert space $\mathscr{H}^{\text {univ }}$ of the entire universe can be factorized in a single way, which defines the preferred factorization. If each atomic quantum system $8^{j}$ is represented by its corresponding Hilbert space $\mathscr{H}^{j}$, then the Hilbert space $\mathscr{H}^{\text {univ }}$ of the universe results

$$
\mathscr{H}^{\text {univ }}=\mathscr{H}^{1} \otimes \mathscr{H}^{2} \otimes \cdots \otimes \mathscr{H}^{j} .
$$

The main appeal of this idea is that it would be in consonance with the standard model of particle physics, where the fundamental blocks of nature are the elemental particles, for example, quarks, electrons, photons, and so forth, and their interactions.

The property ascription to the atomic quantum systems in the AMI follows the general idea of the traditional modal interpretations, where the ascription depends on the state of the system (see [12]). According to standard quantum mechanics, if $\rho^{j}$ is the state of the atomic quantum system $8^{j}$, then the probability that some projector $\Pi_{a}^{j}$ in $8^{j}$ has value 1 is $p\left(\Pi_{a}^{j}\right)=\operatorname{Tr}^{j}\left(\rho^{j} \Pi_{a}^{j}\right)$. The property ascription rule of the AMI assigns the value 1 to the projector $\Pi_{a}^{j}$ when that probability is $p\left(\Pi_{a}^{j}\right)=\operatorname{Tr}^{j}\left(\rho^{j} \Pi_{a}^{j}\right)=1$, that is, if and only if $\Pi_{a}^{j}$ is an eigenprojector of $\rho^{j}$.

In turn, the property ascription for a non-atomic quantum system $8^{\sigma}$ is defined by means of the reduced states of its atomic subsystems. In fact, if the composite quantum system $8^{\sigma}$ consists of the atomic quantum subsystems $8^{1}, 8^{2}, \ldots, 8^{n}$, and $\rho^{\sigma}$ is the state of $\delta^{\sigma}$, then we can compute the reduced states of the atomic subsystems by applying partial traces on $\rho^{\sigma}$, and the eigenprojectors of those reduced states are $\Pi_{i}^{1}, \ldots, \Pi_{j}^{n}$, respectively. The property ascription rule of the AMI for $\varsigma^{\sigma}$ assigns a definite value 1 to the projector $\Pi_{\alpha}^{\sigma}=\Pi_{a}^{1} \otimes \Pi_{b}^{2} \otimes \cdots \otimes \Pi_{d}^{n}$ if and only if the property ascriptions to the atomic quantum subsystems $8^{1}, \wp^{2}, \ldots, \wp^{n}$ assign simultaneously a definite value 1 to the projectors $\Pi_{a}^{1}, \Pi_{b}^{2}, \ldots, \Pi_{d}^{n}$. And since the atomic quantum systems are mutually disjoint, one can also compute the probability that the projector $\Pi_{\alpha}^{\sigma}$ of $8^{\sigma}$ has value 1 , that is, the joint probability that all the projectors $\Pi_{a}^{1}, \Pi_{b}^{2}, \ldots, \Pi_{d}^{n}$ have the value 1 , as

$p\left(\Pi_{\alpha}^{\sigma}\right)=p\left(\Pi_{a}^{1}, \Pi_{b}^{2}, \ldots, \Pi_{d}^{n}\right)=\operatorname{Tr}^{\sigma}\left(\rho^{\sigma} \Pi_{a}^{1} \otimes \Pi_{b}^{2} \otimes \cdots \otimes \Pi_{d}^{n}\right)$.

This implies that the properties of composite non-atomic quantum systems can be deduced from the properties of their atomic subsystems. In mathematical terms, this means that the algebra of properties for a composite system is just the Cartesian product of the algebras of properties for the component atomic subsystems (see [4]).

The main challenge for the AMI is to justify the assumption that there is a preferred partition of the universe, and to provide some idea about how such factorization should look like. Nevertheless, AMI also leads to a conceptual problem. In this interpretation, a non-atomic quantum system $\varsigma^{\sigma}$, defined as composite of atomic quantum systems, does not necessarily have the properties that measurement in quantum mechanics predicts. The reason is that the system $8^{\sigma}$ might be in the quantum state $\rho^{\sigma}$ with an eigenprojector $\Pi^{\sigma}$ such that $\operatorname{Tr}^{\sigma}\left(\rho^{\sigma} \Pi^{\sigma}\right)=1$. This implies that if one measured the property represented by $\Pi^{\sigma}$, one would obtain a positive outcome with probability 1 . But it may be the case that the projector $\Pi^{\sigma}$ is not composite of atomic properties and, therefore, according to the AMI, it is not a property possessed by the composite quantum system $8^{\sigma}$.

Two answers to this conceptual problem have been proposed. The first one was given by Clifton [13], who admits the existence of dispositional properties, that is, properties that may have probability 1 but, nevertheless, do not reveal in measurements. According to this view, reality has a dual description, since there are properties that the system actually possesses and dispositional properties that the system might possess without revealing them.

The second answer is that proposed by Dieks [14], who claims that the projection $\Pi^{\sigma}$ of the composite system $f^{\sigma}$ shows that $f^{\sigma}$ has a collective dynamical effect onto the measurement device, that is, an effect that cannot be explained by the action of the atomic components. In other words, the composite quantum system, when interacting with its environment, can behave as a collective entity, screening the contribution of the atomic quantum systems. This means that sometimes a non-atomic quantum systems $8^{\sigma}$ may be taken as if it were an atomic quantum system, which is equivalent to a coarse-grained description. Dieks argues that this is an everyday exercise in physics where, for certain descriptions of physical processes, it is necessary to take into account not all the components of the system, 
but rather only the emergent properties. This happens, for instance, in the interaction between matter and radiation: in order to explain the absorption and emission of photons in a molecule, one does not need to describe in great detail the properties of all the atoms in this molecule, but only has to give an account of the collective properties. This shows that, in the context of the theory of radiation, the molecule is in itself an atomic entity.

As we will see in the following sections, the MHI supplies a different viewpoint for addressing the question of the ascription of properties to quantum systems, a viewpoint that does not have to face these just explained difficulties.

\section{The Modal-Hamiltonian Interpretation}

Our MHI of quantum mechanics (see [5-10]) also belongs to the modal family, and, therefore, it is also a realist, non-collapse interpretation according to which the quantum state describes the possible properties of a system but not its actual properties. The main difference between the MHI and previous members of the family relies on the property ascription rule: whereas, in the traditional modal interpretations, the rule depends on the state of the system, in the MHI the rule is defined by the Hamiltonian of the closed system. Therefore, the Hamiltonian acquires a central role, both in the definition of systems and subsystems and in the rule that selects the definite valued observables whose possible values become actual.

If we accept the idea that the physical world is made up of quantum systems, we have to identify them. We can cut out the physical reality in many different and arbitrary ways, but only when a portion of reality does not interact with others, we have a nonarbitrary, objective criterion to identify that portion as a system. For this reason, we have designed our interpretation to account for those pieces of reality non-interacting with other pieces, and, so, we have conceived only closed systems as quantum systems. On this basis, and by adopting an algebraic perspective, a quantum system is defined as the following.

Systems Postulate (SP). A quantum system $\&$ is represented by a pair $(\mathcal{O}, H)$ such that (i) $\mathcal{O}$ is a space of self-adjoint operators on a Hilbert space $\mathscr{H}$, representing the observables of the system, (ii) $H \in \mathcal{O}$ is the time-independent Hamiltonian of the system $\mathcal{S}$, and (iii) if $\rho_{0} \in \mathcal{O}^{\prime}$ (where $\mathcal{O}^{\prime}$ is the dual space of $\mathcal{O}$ ) is the initial state of $S$, it evolves according to the Schrödinger equation in its von Neumann version.

Of course, any quantum system can be partitioned in many ways; however, not any partition will lead to parts which are, in turn, quantum systems (see $[15,16])$. Then, a composite system is defined as the following.

Composite Systems Postulate (CSP). A quantum system represented by $\&:(\mathcal{O}, H)$, with initial state $\rho_{0} \in \mathcal{O}^{\prime}$, is composite when it can be partitioned into two quantum systems $\mathcal{f}^{1}:\left(\mathcal{O}^{1}, H^{1}\right)$ and $\mathcal{f}^{2}:\left(\mathcal{O}^{2}, H^{2}\right)$ such that (i) $\mathcal{O}=$ $\mathcal{O}^{1} \otimes \mathcal{O}^{2}$ and (ii) $H=H^{1} \otimes I^{2}+I^{1} \otimes H^{2}$, (where $I^{1}$ and $I^{2}$ are the identity operators in the corresponding tensor product spaces). In this case, the initial states of $\delta^{1}$ and $\delta^{2}$ are obtained as the partial traces $\rho_{0}^{1}=\operatorname{Tr}_{(2)} \rho_{0}$ and $\rho_{0}^{2}=\operatorname{Tr}_{(1)} \rho_{0}$; we say that $S^{1}$ and $S^{2}$ are subsystems of the composite system, $s=\delta^{1} \cup \delta^{2}$. If the system is not composite, it is elemental.

From this viewpoint, a composite system $\delta$, composed by two non-interacting elemental systems $\delta^{1}$ and $\delta^{2}$, may become an elemental system $\delta^{\prime}$ from the time $t_{1}$ at which the interaction between $\delta^{1}$ and $\delta^{2}$ begins, If, at a later time $t_{2}$ the interaction ends, the system $\delta^{\prime}$ may become the original composite system 8 , composed again by the elemental systems $\delta^{1}$ and $\delta^{2}$. If the interaction during the time interval $t_{2}-t_{1}$ results in the entanglement between the states $\rho^{1}$ of $\delta^{1}$ and $\rho^{2}$ of $\delta^{2}$, there will be quantum correlations between $\delta^{1}$ and $\delta^{2}$. In other words, according to the MHI, two systems may be nonlocally correlated if they interacted in the past although at present they do not longer interact and, as a consequence, they are elemental.

Since the contextuality of quantum mechanics, as implied by the Kochen-Specker theorem (see [11]), prevents us from consistently assigning actual values to all the observables of a quantum system in a given state, the second step is to identify the preferred context, that is, the set of the actual-valued observables of the system. Whereas the different rules of actual-value ascription proposed by previous modal interpretations rely on mathematical properties of the theory, our MHI places an element with a clear physical meaning, the Hamiltonian, at the heart of its rule.

Actualization Rule (AR). Given an elemental quantum system represented by $\&:(\mathcal{O}, H)$, the actual-valued observables of $\delta$ are $H$ and all the observables commuting with $H$ and having, at least, the same symmetries as $H$.

The MHI preferred context where actualization occurs is independent of time; the actual-valued observables always commute with the Hamiltonian, and, therefore, they are constants of motion of the system. In other words, the observables that receive actual values are the same during all the "life" of the quantum system as such-precisely, as a closed system-: there is no need of accounting for the dynamics of the actual properties of the quantum system as in other modal interpretations (see [17]).

The fact that the Hamiltonian always belongs to the preferred context agrees with the many physical cases where the energy has definite value. The MHI has been applied to several well-known physical situations (hydrogen atom, Zeeman effect, fine structure, etc.), leading to results consistent with experimental evidence (see [5]). Moreover, it has proved to be effective not only for solving the measurement problem in its ideal versions, but also for overcoming the deep challenges that non-ideal measurements pose to other modal interpretations (see $[18,19])$. In particular, the MHI distinguishes between reliable and nonreliable non-ideal measurements (see [5]). Furthermore, in spite of the fact that MHI applies to closed systems, we have proved its compatibility with environment-induced decoherence (see $[20,21])$.

Once the MHI was clearly formulated, our further question was whether it satisfies the Galilei invariance of quantum mechanics. In fact, any continuous transformation 
admits two interpretations. Under the active interpretation, the transformation corresponds to a change from one system to another transformed system; under the passive interpretation, the transformation consists in a change of the viewpoint reference frame from which the system is described (see [22]). Nevertheless, in both cases, the validity of a group of symmetry transformations expresses the fact that the identity and the behavior of the system are not altered by the application of the transformations: in the active interpretation language, the original and the transformed systems are equivalent; in the passive interpretation language, the original and the transformed reference frames are equivalent. Then, any realist interpretation should agree with that physical fact: the rule of actual-value ascription should select a set of actual-valued observables that remains unaltered under the transformations. Since the Casimir operators of the central-extended Galilei group are invariant under all the transformations of the group, one can reasonably expect that those Casimir operators belong to the preferred context.

As we have seen, the preferred context selected by AR only depends on the Hamiltonian of the system. Then, the requirement of invariance of the preferred context under the Galilei transformations is directly fulfilled when the Hamiltonian is invariant, that is, in the case of time displacement, space displacement, and space rotation,

$$
\begin{array}{ll}
H^{\prime}=e^{i H \tau} H e^{-i H \tau}=H & (\text { since }[H, H]=0), \\
H^{\prime}=e^{i P_{i} r_{i}} H e^{-i P_{i} r_{i}}=H & \left(\text { since }\left[P_{i}, H\right]=0\right), \\
H^{\prime}=e^{i J_{i} \theta_{i}} H e^{-i J_{i} \theta_{i}}=H \quad\left(\text { since }\left[J_{\mathrm{i}}, H\right]=0\right) .
\end{array}
$$

However, it is not clear that the requirement is completely satisfied, since the Hamiltonian is not invariant under Galilei boosts. In fact, under a Galilei boost corresponding to a velocity $u_{x}, H$ changes as

$$
H^{\prime}=e^{i K_{x}^{(G)} u_{x}} H e^{-i K_{x}^{(G)} u_{x}} \neq H \quad\left(\text { since }\left[K_{x}^{(G)}, H\right]=i P_{x} \neq 0\right) .
$$

Nevertheless, when space is homogeneous and isotropic, a Galilei boost only introduces a change in the subsystem that carries the kinetic energy of translation; the internal energy $W$ remains unaltered under the transformation. This should not sound surprising to the extent that $W$-multiplied by $m$-is a Casimir operator of the central-extended Galilei group. On this basis, we can reformulate AR in an explicit Galilei-invariant form in terms of the Casimir operators of the central-extended group.

Actualization Rule' (AR'). Given an elemental quantum system free from external fields and represented by 8 : $(\mathcal{O}, H)$, its actual-valued observables are the observables $C_{i}^{G}$ represented by the Casimir operators of the centralextended Galilei group in the corresponding irreducible representation, and all the observables commuting with the $C_{i}^{G}$ and having, at least, the same symmetries as the $C_{i}^{G}$.

Since the observables $C_{i}^{G}$-in the reference frame of the center of mass-are $M, m W$, and $m^{2} S^{2}$, this reformulation $A R$ ' is in agreement with the original AR when applied to a system free from external fields (see $[7,9])$. (i) The actual valuedness of $M$ and $S^{2}$, postulated by AR', follows from AR; these observables commute with $H$ and do not break its symmetries because, in nonrelativistic quantum mechanics, both are multiples of the identity in any irreducible representation.

(ii) The actual valuedness of $W$ might seem to be in conflict with AR because $W$ is not the Hamiltonian, whereas $W$ is Galilei invariant, and $H$ changes under the action of a Galilei boost. However, this is not a real obstacle because a Galilei-boost transformation only introduces a change in the subsystem that carries the kinetic energy of translation, which can be considered a mere shift in an energy defined up to a constant (see $[7,9])$.

Summing up, the application of AR' leads to reasonable results, since the actual-valued observables turn out to be invariant and, therefore, objective magnitudes. The assumption of a strong link between invariance and objectivity is rooted in a natural idea; what is objective should not depend on the particular perspective used for the description; or, in group-theoretical terms, what is objective according to a theory is what is invariant under the symmetry group of the theory. This idea is not new; it was widely discussed in the context of special and general relativity with respect to the ontological status of space and time (see [23]), and it reappeared in several works (see [24-27]). From this perspective, AR' says that the observables that acquire actual values are those representing objective magnitudes. On the other hand, from any realist viewpoint, the fact that certain observables acquire an actual value is an objective fact in the behavior of the system; therefore, the set of actualvalued observables selected by a realist interpretation must be also Galilei invariant. But the Galilei-invariant observables are always functions of the Casimir operators of the Galilei group. As a consequence, one is led to the conclusion that any realist interpretation that intends to preserve the objectivity of actualization may not stand very far from the MHI.

\section{The "Atomic" Systems in the MHI}

On the basis of the two previous sections, it is quite clear that the AMI and the MHI, although both belonging to the modal family, are very different approaches to the problem of the interpretation of quantum mechanics. Nevertheless, when analyzed in the light of the basic assumptions of the AMI, the $\mathrm{MHI}$ can also be viewed as a kind of "atomic" interpretation, in two different senses.

4.1. Factorization in Elemental Systems. As we have seen, the AMI supposes that there are certain building blocks in nature, the atomic systems, in such a way that the universe is built by those fundamental blocks. This means that there is a preferred factorization of the universe, and any non-atomic quantum systems is a composite system whose components are the atomic systems. Therefore, the ascription of properties to the composite systems is determined by the properties ascribed to its atomic components. 
When the main idea of the AMI is expressed in these simple terms, the same characterization can be applied to the MHI, which, as a consequence, can be considered as an atomic interpretation. In fact, the composite systems postulate CSP states that a composite system $\&$, represented by its space of observables $\mathcal{O}$, can be factorized into its non-interacting components $f^{A}$ and $\delta^{B}$ in such a way that $\mathcal{O}=\mathcal{O}^{A} \otimes \mathcal{O}^{B}$. It is not difficult to see that the recursive application of this postulate to the universe as a whole, then to its components $\delta^{A}$ and $\delta^{B}$, then to the components of these components and so on, will finally lead to a set of systems $8^{1}, f^{2}, \ldots, 8^{n}$, which cannot be further decomposed: these systems are not called "atomic" systems in the MHI, but "elemental" systems, and they also play the role of fixing the preferred factorization of the universe

$$
\mathcal{O}^{\text {univ }}=\mathcal{O}^{1} \otimes \mathcal{O}^{2} \otimes \cdots \otimes \mathcal{O}^{j} .
$$

Moreover, although the MHI is formulated from the algebraic perspective, it can also be expressed in the Hilbert space language of the AMI. According to the algebraic formalism of quantum mechanics (see [28]), given a ${ }^{*}$-algebra $\mathcal{A}$ of operators, (i) the set of the self-adjoint elements of $\mathcal{A}$ is the space $\mathcal{O}$, whose elements represent observables, $O \in \mathcal{O}$, and (ii) states are represented by functionals on $\mathcal{O}$, that is, by elements of the dual space $\mathcal{O}^{\prime}, \rho \in \mathcal{O}^{\prime}$. The traditional presentations of the algebraic formalism adopt a $C^{*}$-algebra of operators as the departing point: a $\mathrm{C}^{*}$-algebra can be represented by a Hilbert space (GNS theorem) and, in this particular case, $\mathcal{O}=\mathcal{O}^{\prime}$; therefore, $\mathcal{O}$ and $\mathcal{O}^{\prime}$ are both represented by $\mathscr{H} \otimes \mathscr{H}$. As a consequence, in the Hilbert space formalism, the factorization into elemental systems introduced by the MHI can also be expressed (see (1)) as

$$
\mathscr{H}^{\text {univ }}=\mathscr{H}^{1} \otimes \mathscr{H}^{2} \otimes \cdots \otimes \mathscr{H}^{j} .
$$

As we have pointed out in Section 3, a composite system may become an elemental system from the time at which the interaction between its elemental component subsystems begins. As a consequence, the factorization of the universe into elemental systems introduced by the MHI is not time independent but changes with time. Nevertheless, this is not a shortcoming of the interpretation to the extent that, according to the MHI, the elemental systems resulting from this preferred factorization are not the building blocks of the universe; as we will discuss in detail in the next subsection, this role is played by the atomic irreducible systems.

On the other hand, in the MHI, the ascription of properties to the composite systems is also determined by the properties ascribed to its atomic components. In fact, the actualization rule AR applies to elemental quantum systems. In turn, the original detailed formulation of the MHI (see [5]) includes a precise interpretative postulate that establishes the links between the properties of a composite system and the properties of its components.

Composite Properties Postulate (CPP). Given a composite quantum system $\delta=\wp^{1} \cup \delta^{2}:(\mathcal{O}, H)$, where $\AA^{1}:\left(\mathcal{O}^{1}, H^{1}\right)$ and $8^{2}:\left(\mathcal{O}^{2}, H^{2}\right)$, and given the observables $A^{1} \in \mathcal{O}^{1}$ of $\AA^{1}, A^{2} \in \mathcal{O}^{2}$ of $\AA^{2}$, and the observables $A^{1} \otimes I^{2} \in \mathcal{O}$ and $A^{f}=f\left(A^{1} \otimes I^{2}, I^{1} \otimes A^{2}\right) \in \mathcal{O}$ of $s$, where $f$ is an analytical function, then, (i) the observables $A^{1}$ and $A^{1} \otimes I^{2}$ represent the same property with values $a_{i}^{1}$, where the $a_{i}^{1}$ are the eigenvalues of both $A^{1}$ and $A^{1} \otimes I^{2}$, and (ii) the observable $A^{f}$ represents a property with values $f\left(a_{i}^{1}, a_{j}^{2}\right)$, where the $a_{i}^{1}, a_{j}^{2}$ are the eigenvalues of $A^{1}$ and $A^{2}$, respectively; $A^{f}$ is equivalent to the combination between $A^{1}$ and $A^{2}$, represented by the function $f$.

The interpretational postulate CPP expresses the usual quantum assumption according to which the observable $A^{1}$ of a subsystem $\delta^{1}$ and the observable $A^{1} \otimes I^{2}$ of the composite system $\delta=\delta^{1} \cup \delta^{2}$ represent the same property. On the other hand, this postulate establishes the necessary connections between the properties of the composite system and the properties of its subsystems. The assumption of these connections is not a specific feature of quantum mechanics but is also usual in classical mechanics where we consider, for instance, the energy of a two-particles composite system as a particular combination (expressed by the sum) of the energies of the component subsystems.

Let us recall that the original AMI also leads to a conceptual problem when the property ascription to atomic systems is compared with the supposed results of measurements. Our MHI undercuts that problem from the very beginning by supplying a detailed account of quantum measurements, which takes into account that their final goal is not to "discover" the actual value of a system's observable, but to reconstruct the state of the system just before the beginning of the measurement process. Therefore, the only relevant fact is the definite reading of the apparatus' pointer; the task consists in explaining how the repetition of single detections where the pointer is definite valued allows us to reconstruct the state of the measured system. According to the MHI, no matter what happens with the measured system, in each single measurement, the apparatus' pointer is always definite valued because its Hamiltonian commutes with its pointer. And this commutation relation is not required by the interpretation, but by the fact that, for the reading of the pointer to be possible, the eigenvectors of the pointer have to be stationary; thus, the apparatus has to be constructed in such a way that its pointer commutes with its Hamiltonian (we refer the reader to [5]).

On the basis of this account of the quantum measurement, the definite value of the measurement apparatus is explained both in the ideal and in the non-ideal cases, and this treatment even allows us to distinguish between reliable and nonreliable non-ideal measurements (see Section 6.2 of [5]). Moreover, we have also shown the compatibility between the MHI account of measurement and the explanations given in the context of the theory of the environmentinduced decoherence (see $[20,21])$. Therefore, in the MHI framework there is no inconsistency between the property ascription to elemental systems and the properties effectively detected as definite valued in measurements.

Summing up, in a relevant sense, the MHI can be conceived as a case of AMI, but, with an important advantage: by contrast to the original AMI, the MHI provides a precise criterion of factorization. Such a criterion is given by the 
Hamiltonian of the whole universe, and the possibility of decomposing it into non-interacting elemental Hamiltonians. The justification of this criterion is not based on a priori arguments or principles, but on the fruitfulness of the MHI itself, which relies on the physical relevance of the interpretation when applied to well-known physical situations and on its capability of solving some traditional interpretational challenges. In turn, once the actualization rule is applied to the elemental systems, the assignment of properties to any composite system is derived from the equivalence between its properties and the properties of its component subsystems on the basis of the composite properties postulate CPP. This bottom-up "constructive" strategy for assigning properties is a further point of agreement between the MHI and the AMI.

4.2. Atomic Irreducible Systems. In the previous subsection we have seen a close analogy between the roles played by the atomic systems in the AMI and the elemental systems in the MHI. Nevertheless, the MHI also incorporates conceptual resources to think about of atomic systems in a new sense. These resources come from the theoretical perspective supplied by group theory.

As stressed by Lévi-Leblond (see [29]), although it is usual to read that non-relativistic quantum mechanics is covariant, and even invariant, under the Galilei transformations, this issue has been scarcely treated in the standard literature on the theory. For instance, the commutation relations defining the Galilei group are often not even quoted in the textbooks on the matter (an exception is [30]). This situation has its counterpart in the field of the interpretation of quantum mechanics; the relevance of the Galilei group is rarely discussed in the impressive amount of literature on the subject.

By contrast with this trend, our MHI places the Galilei group at the very heart of the interpretation. As we have seen, when the actualization rule is expressed under a Galilei invariant form, the properties that acquire definite values are those represented by the Casimir operators of the Galilei group (see [7, 9]). Besides endowing the actualvalued observables with the objectivity required by any realist interpretation, this group-theoretical approach allows us to identify atomic systems in a sense different from that underlying the concept of elemental system. In fact, in complete agreement with the assumptions of quantum field theory, we can also say in non-relativistic quantum mechanics that the atomic systems are the elemental particles, which are represented by the irreducible representations of the Galilei group.

Let us notice the difference between an elemental system and an atomic-irreducible system, which can be captured by the MHI. The atomic systems are the final building blocks of the universe, in the sense that there is no simpler system that can exist as an individual entity in the quantum world. When two or more atomic systems do not interact, the resulting system is trivially composite since the atomic systems still persist as such. But when two or more atomic systems interact with each other, the result is a new system where the original components do not preserve their individuality; the resulting system may be elemental in the sense that cannot be further decomposed into independently evolving subsystems.

The original AMI cannot stress this difference because it does not consider the difference between the non-interacting and the interacting cases: in the AMI framework, the problem is always the question about the factorization of the Hilbert space, without taking into account that such a factorization does not always represent physical independence between subsystems. For this reason, whereas the AMI interprets the atomic systems as representing the elemental particles, at the same time Dieks admits that, in the interaction between matter and radiation, the molecule in itself can be treated as an atomic entity. However, Dieks' claim sounds dissonant when one expects that the atomic systems play the role of the building blocks of the universe; a molecule is not an elemental particle. But when we take into account interactions, we can say that the elemental particles are the atoms, the building blocks of reality, and at the same time we can explain that a molecule, being a non-atomic system, is nevertheless an elemental system that can no longer be decomposed into independent subsystems whose evolution is ruled by the dynamical postulate of quantum mechanics.

\section{Decomposition of the Hilbert Space}

As we have stressed in the previous section, the MHI provides a precise criterion for the factorization of the Hilbert space of a composite system into the Hilbert spaces of its elemental subsystems. In this section, we will see that the MHI also introduces a partition of the Hilbert space of any elemental system, which determines what observables acquire definite actual values.

As it is well known, any observable can be described in terms of its spectral decomposition, where each eigenprojector projects onto a subspace of a Hilbert space, which may be degenerate or not; each one of those subspaces corresponds to a definite eigenvalue of the observable. Formally, let us consider the space of the observables $\mathcal{O}$ acting on the Hilbert space $\mathcal{H}$. Let us also consider a certain observable $A \in \mathcal{O}$, which can be written in terms of its spectral decomposition as

$$
A=\sum_{n, i_{n}} a_{n}\left|n, i_{n}\right\rangle\left\langle n, i_{n}\right|
$$

where $a_{n}$ is the eigenvalue associated to the eigenvectors $\left|n, i_{n}\right\rangle, a_{n} \neq a_{n^{\prime}}$, the index $n$ corresponds to the eigenvalue, and the index $i_{n}$ corresponds to the degeneracy of each eigenvalue. Then, an equivalence relation between eigenvectors corresponding to the same eigenvalue can be defined as follows

Definition 1. Two vectors $|a\rangle$ and $|b\rangle$ are equivalent with respect to an observable $A,|a\rangle \underset{A}{\sim}|b\rangle$, if $A|a\rangle=a_{n}|a\rangle$ and $A|b\rangle=a_{n}|b\rangle$.

This means that $|a\rangle$ and $|b\rangle$ are equivalent with respect to the observable $A$ if they are eigenvectors of the same eigenvalue $a_{n}$. When the eigenvalue is not degenerate, the only possibility is that $|a\rangle=c|b\rangle$; equivalent vectors are 
colinear. If the eigenvalue is degenerate, then the relation implies that all the vectors belonging to the degenerate subspace associated to $a_{n}$ are equivalent. This equivalence relation introduces a decomposition of the Hilbert space into the eigensubspaces $\left[A_{n}\right]$ of the observable $A$, such that for any $|a\rangle,|b\rangle \in\left[A_{n}\right],|a\rangle \underset{A}{\sim}|b\rangle$. Moreover, given the subspace $\left[A_{n}\right]$, the subspace $\neg\left[A_{n}\right]$ can be defined as the orthogonal complement of the subspace corresponding to the eigenvalue $a_{n}$. Then, for each eigenvalue $a_{n}$, the Hilbert space $\mathcal{H}$ can be decomposed into two disjoint and exhaustive subspaces $\left[A_{n}\right]$ and $\neg\left[A_{n}\right]$, where $\neg\left[A_{n}\right]$ is subspace corresponding to the remaining eigenvalues of the observable $A$.

Among all the decomposition of the system's Hilbert space, there are two extreme cases. One of them is the case of any nondegenerate observable, say $B$, whose spectral decomposition is given by

$$
B=\sum_{m} b_{m}|m\rangle\langle m|
$$

where $b_{m} \neq b_{m^{\prime}}$ and $\{|m\rangle\}$ is a basis of the Hilbert space $\mathcal{H}$. Here $B$ decomposes $\mathcal{H}$ into its unidimensional subspaces. The other extreme case is the the completely degenerate observable, that is, the identity $I$, which has only one eigenvalue; therefore, a single subspace $[I]$ can be defined, which is precisely the whole $\mathcal{H}$.

From the perspective of the formal structure of quantum mechanics, all the observables are on the same footing. However, from a physical viewpoint, there is a substantial fact that makes the Hamiltonian different from the remaining observables of the system; it is the observable that rules the evolution of the quantum systems through the dynamical law of the theory. The MHI takes the central role played by the Hamiltonian in the theory and translates it to interpretation. Therefore, here we are interested in the decomposition of the Hilbert space introduced by the Hamiltonian.

Let us consider an elemental quantum system represented by $\&:(\mathcal{O}, H)$, where $\mathcal{O}=\mathscr{H} \otimes \mathscr{H}$ and $M=\operatorname{dim}(\mathscr{H})$. Let us also suppose that the Hamiltonian $H$ has a discrete spectrum, and, in the generic case, it is degenerate; then, it can be expressed as

$$
H=\sum_{n=1}^{N} \omega_{n} \Pi_{n},
$$

where $\omega_{n} \neq \omega_{n^{\prime}}$ and $N$ is the number of different eigenvalues of $H$. Each projector $\Pi_{n}$, corresponding to the eigenvalue $\omega_{n}$, projects onto its corresponding eigensubspace $\left[H_{n}\right]$ resulting from the decomposition of $\mathscr{H}$ introduced by $H$ and can be expressed as

$$
\Pi_{n}=\sum_{i_{n}=1}^{f(n)}\left|n, i_{n}\right\rangle\left\langle n, i_{n}\right|,
$$

where the index $i_{n}$ expresses the degeneracy of the energy eigenvalue $\omega_{n}$, and $f(n)$ is the dimension of the eigensubspace $\left[H_{n}\right]$. According to the actualization rule AR, the actual-valued observables of the system $\&$ are those commuting with the Hamiltonian $H$ and having, at least, the same symmetries as $H$. Therefore, those observables have the following form:

$$
A=\sum_{n=1}^{N} a_{n} \Pi_{n}=\sum_{n=1}^{N} a_{n} \sum_{i_{n}=1}^{f(n)}\left|n, i_{n}\right\rangle\left\langle n, i_{n}\right| .
$$

In fact, it is clear that $[A, H]=0$. Moreover, if $a_{n} \neq a_{n^{\prime}}$, $A$ has the same degeneracy as $H$ since they have the same eigenprojectors; the eigensubspace $\left[A_{n}\right]$ spanned by the eigenvectors corresponding to the degenerate eigenvalue $a_{n}$ of $A$ is the same as the eigensubspace $\left[H_{n}\right]$ spanned by the eigenvectors corresponding to the degenerate eigenvalue $\omega_{n}$. In turn, if $a_{n} \neq a_{n^{\prime}}$ does not hold, then the observable $A$ has more symmetries than $H$ since it does not distinguish between certain eigensubspaces $\left[H_{n}\right]$ resulting from the decomposition of $\mathscr{H}$ introduced by $H$. On the other hand, any observable of the form

$$
B=\sum_{n=1}^{N} b_{n, i_{n}} \Pi_{n}=\sum_{n=1}^{N} b_{n, i_{n}} \sum_{i_{n}=1}^{f(n)}\left|n, i_{n}\right\rangle\left\langle n, i_{n}\right|
$$

will not acquire an actual value, in spite of commuting with $H$. This is due to the fact that the observable $B$, through its eigenvalues, would discriminate among the eigenvectors of $H$ corresponding to a single degenerate eigenvalue of $H$. In other words, $B$ would distinguish among vectors belonging to the same eigensubspace $\left[H_{n}\right]$ resulting from the decomposition of $\mathcal{H}$ introduced by $H$, that is, among vectors that are equivalent with respect to $H$.

Summing up, besides defining a preferred factorization of the universe into elemental quantum systems, the MHI introduces a preferred decomposition of the Hilbert space of each elemental system into subspaces. In this way, the interpretation identifies with precision the definite actualvalued observables of the system (and it also gives the number of the different kinds of those observables, see the Appendix). The preferred decomposition can be viewed as a coarse graining of the Hilbert space; no observable whose actualization would discriminate among eigenvectors not distinguished by the Hamiltonian can become actual. It is precisely this coarse-grained decomposition what identifies with precision the observables that acquire a definite actual value according to our interpretation.

\section{Conclusions}

The modal interpretations of quantum mechanics were designed to solve the measurement problem from a realist perspective without the collapse hypothesis. Among them, the AMI intended to accomplish that task without breaking the Kochen-Specker restrictions, by postulating the existence of a preferred factorization of the Hilbert space of the universe. According to the AMI, such a factorization defines the Hilbert spaces representing the atomic systems that populate the quantum reality; those atomic systems are conceived as the building blocks of the universe, that is, the elemental particles postulated by physics. However, its main 
problem was the fact that it did not supply a precise criterion for that factorization; for this reason, the AMI did not have as much relevance as other modal interpretations.

In this paper, we have compared our recently presented MHI with the AMI, in order to show that the MHI can be viewed as a kind of atomic interpretation that overcomes the main difficulty of the original AMI by precisely identifying the preferred factorization. Nevertheless, the systems defined by that factorization are not "atoms", but elemental systems that cannot be further decomposed in quantum systems independently evolving according to the Schrödinger equation. Those elemental systems may be the result of the interaction of atomic systems conceived as elemental particles. Moreover, the MHI also provides a precise criterion for defining those atomic systems, the elemental particles, which can be viewed as the building blocks of the universe; they are the systems represented by the irreducible representations of the Galilei group. On the other hand, on the basis of the decomposition of the Hilbert space of a system into the eigensubspaces of the Hamiltonian, the MHI also unequivocally identifies the observables that acquire definite actual values in any elemental system. The original AMI cannot introduce all these distinctions because it does not take into account the great relevance of the interactions in the quantum world.

Finally, we want to stress that the central role conferred to group theory by the MHI allows us to expect that the interpretation can be transferred to quantum field theory by replacing the Galilei group with the Poincaré group plus the group of the internal symmetries. At present, this is a work in progress (see $[31,32])$.

\section{Appendix}

Let us consider a system $\delta:(\mathcal{O}, H)$ whose Hamiltonian $H$ has a discrete spectrum with $N$ different eigenvalues and, therefore, $N$ eigensubspaces $\left[H_{n}\right]$ that may be degenerate or not. The dimension of the Hilbert space $\mathscr{H}$ is $M=$ $\operatorname{dim}(\mathscr{H})=\sum_{i=1}^{N} \operatorname{dim}\left(\left[H_{n}\right]\right)$. Then, $\mathscr{H}$ can be decomposed in $C(M)$ ways according to the following equation:

$$
M=1 f_{1}^{(M)}(k)+2 f_{2}^{(M)}(k)+\cdots+N f_{M}^{(M)}(k)=\sum_{j=1}^{M} j f_{j}^{(M)}(i)
$$

where $j=1,2, \ldots, M$ are the different dimensions that the Hamiltonian's eigensubspaces $\left[H_{n}\right]$ may have, $f_{j}^{(M)}$ is the number of eigensubspaces of dimension $j$, and $i$ denotes the particular solution of (A.1). The number $C(M)$ is the number of solutions of (A.1).

Let us see an example with $M=\operatorname{dim}(\mathscr{H})=4$; in this case, (A.1) reads

$$
4=1 f_{1}^{(4)}+2 f_{2}^{(4)}+3 f_{3}^{(4)}+4 f_{4}^{(4)} .
$$

There are five possible solutions to this equation, and, therefore, $C(4)=5$. If each solution is denoted by $g^{(M)}(i)$, then

$$
\begin{aligned}
& g^{(4)}(1)=\left\{f_{1}^{(4)}(1)=4, f_{2}^{(4)}(1)=0, f_{3}^{(4)}(1)=0, f_{4}^{(4)}(1)=0\right\}, \\
& g^{(4)}(2)=\left\{f_{1}^{(4)}(2)=2, f_{2}^{(4)}(2)=1, f_{3}^{(4)}(2)=0, f_{4}^{(4)}(2)=0\right\}, \\
& g^{(4)}(3)=\left\{f_{1}^{(4)}(3)=1, f_{2}^{(4)}(3)=0, f_{3}^{(4)}(3)=1, f_{4}^{(4)}(3)=0\right\}, \\
& g^{(4)}(4)=\left\{f_{1}^{(4)}(4)=0, f_{2}^{(4)}(4)=2, f_{3}^{(4)}(4)=0, f_{4}^{(4)}(4)=0\right\}, \\
& g^{(4)}(5)=\left\{f_{1}^{(4)}(5)=0, f_{2}^{(4)}(5)=0, f_{3}^{(4)}(5)=0, f_{4}^{(4)}(5)=1\right\},
\end{aligned}
$$

where

(i) $g^{(4)}(1)$ corresponds to the decomposition of $\mathscr{H}$ into four one-dimensional eigensubspaces.

(ii) $g^{(4)}(2)$ corresponds to the decomposition of $\mathscr{H}$ into two one-dimensional eigensubspaces and one eigensubspace of dimension 2.

(iii) $g^{(4)}(3)$ corresponds to the decomposition of $\mathscr{H}$ into one one-dimensional eigensubspace and one eigensubspace of dimension 3 .

(iv) $g^{(4)}(4)$ corresponds to the decomposition of $\mathscr{H}$ into two eigensubspaces of dimension 2.

(v) $g^{(4)}(5)$ corresponds to the decomposition of $\mathscr{H}$ into one eigensubspace of dimension 4 .

Let us now suppose for a moment that the eigensubspaces $\left[H_{n}\right]$ of $H$ are all one dimensional. Then, we can compute the number $\operatorname{Obs}(M)$ of different kinds of the observables that can be built from the preferred factorization, that is, of the observables that commute with $H$ and do not break its symmetry. For this purpose, we can use the properties of the permutation group $S_{M}$ of $M$ objects, which can be written in terms of cycles, where a cycle is a cyclic permutation of a subset of the group. An arbitrary element has $f_{j}^{(M)}(i)$ cycles of order $j$, according to (A.1). In turn, in this permutation group, conjugacy classes can be defined, which give the cyclic structure; those classes are labeled by the integer numbers $f_{j}^{(M)}(i)$. The number of elements of each conjugacy class will be the number of possible combinations that can be performed with the decomposition introduced by the Hamiltonian. If each conjugacy class consists of permutations with $f_{1}^{(M)}(i)$ cycles of order $1, f_{2}^{(M)}(i)$ cycles of order 2, and so forth, which satisfy (A.1), then the number $P_{M}$ of different permutations in the conjugacy class is (see [33, page 37 , equation $(1.141)]$ )

$$
P_{M}=\frac{M !}{\prod_{j}(j !)^{f_{j}^{(M)}(i)} f_{j}^{(M)}(i) !} .
$$

Therefore, the number $\mathrm{Obs}(M)$ of different kinds of observables that will acquire definite actual values according the 
actualization rule of our MHI is the sum of all the possible decompositions

$\operatorname{Obs}(M)$

$$
=\sum_{i=1}^{C(M)} \frac{M !}{\left(\operatorname{Max}\left(g^{(M)}(i)\right)-1\right) !\left(\prod_{j=1}^{M}(j !)^{f_{j}^{(M)}(i)} f_{j}^{(M)}(i) !\right)},
$$

where the value $\operatorname{Max}\left(g^{(M)}(i)\right)$ is the maximum dimension of all the eigensubspaces corresponding to the decomposition $g^{(M)}(i)$. The coefficient $\left(\operatorname{Max}\left(g^{(M)}(i)\right)-1\right)$ must be introduced because the permutations of cyclic order inside one conjugacy class should not be counted.

Let us see how (A.5) works in the same example treated above: a Hilbert space of dimension $M=4$. If all the eigenvalues of the Hamiltonian are nondegenerate, there are 4 eigensubspaces $\left[H_{n}\right]$. Then,

$$
\begin{aligned}
\operatorname{Obs}(4) & =\sum_{i=1}^{5} \frac{4 !}{\left(\operatorname{Max}\left(g^{(4)}(i)\right)-1\right) ! \prod_{j=1}^{4}(j !)^{f_{j}^{(4)}(i)} f_{j}^{(4)}(i) !} \\
& =\frac{4 !}{4 !}+\frac{4 !}{2 ! 2 !}+\frac{4 !}{3 !}+\frac{4 !}{2 ! 2 ! 2 !}+\frac{4 !}{4 !} \\
& =1+6+4+3+1=15,
\end{aligned}
$$

where:

(i) $g^{(4)}(1)$ corresponds to the four one-dimensional eigensubspaces of $H$, that is, $H=\left\{\left[H_{1}, H_{2}, H_{3}, H_{4}\right]\right\}$. Therefore, for this decomposition, we have only one kind of observables, represented by the first value 1 in the second member of (A.6).

(ii) $g^{(4)}(2)$ corresponds to the composition of eigensubspaces of $H$ into two one-dimensional subspaces and one subspace of dimension 2 . Therefore, in this case, we obtain 6 different kinds of observables: $\left\{\left[H_{1}, H_{2}, H_{34}\right]\right\},\left\{\left[H_{1}, H_{3}, H_{24}\right]\right\},\left\{\left[H_{1}\right.\right.$, $\left.\left.H_{4}, H_{23}\right]\right\}, \quad\left\{\left[H_{2}, H_{3}, H_{14}\right]\right\}, \quad\left\{\left[H_{2}, H_{4}, H_{13}\right]\right\}$, and $\left\{\left[H_{3}, H_{4}, H_{12}\right]\right\}$. These six kinds of observables are represented by the value 6 in the second member of (A.6).

(iii) $g^{(4)}(3)$ corresponds to the composition of the eigensubspaces of $H$ into one one-dimensional subspace and one subspace of dimension 3. Therefore, in this case, we obtain 4 different kinds of observables: $\left\{\left[H_{1}, H_{234}\right]\right\},\left\{\left[H_{2}, H_{134}\right]\right\},\left\{\left[H_{3}, H_{124}\right]\right\}$, and $\left\{\left[H_{4}, H_{123}\right]\right\}$. These four kinds of observables are represented by the value 4 in the second member of (A.6).

(iv) $g^{(4)}(4)$ corresponds to the composition of the eigensubspaces of $H$ into two subspaces of dimension 2. Therefore, in this case, we obtain 3 different kinds of observables: $\left\{\left[H_{12}, H_{34}\right]\right\},\left\{\left[H_{13}, H_{24}\right]\right\}$, and $\left\{\left[H_{14}, H_{23}\right]\right\}$. These three kinds of observables are represented by the value 3 in the second member of (A.6). (v) $g^{(4)}(5)$ corresponds to the composition of the eigensubspaces of $H$ into only one subspace of dimension 4. The only kind of observables is given by $\left[H_{1234}\right]=$ $\mathcal{H}$, which defines the observables that are multiples of the identity. This kind is represented by the second value 1 in the second member of (A.6).

Up to now, we have considered the case where all the Hamiltonian's eigenvalues are not degenerate, and, then, the eigensubspaces $\left[H_{n}\right]$ of $H$ are all one dimensional. However, the strategy can be extrapolated to a general case. For instance, let us suppose that $H$ has a single degenerate eigenvalue, say, that corresponding to the subspace $\left[\mathrm{H}_{34}\right]$. In this case, we can rename $\left[H_{1}\right] \rightarrow\left[H_{1}\right]^{\prime},\left[H_{2}\right] \rightarrow\left[H_{2}\right]^{\prime}$, and $\left[\mathrm{H}_{34}\right] \rightarrow\left[\mathrm{H}_{3}\right]^{\prime}$, and we can repeat the computation as if we were working with a Hilbert space of dimension 3.

\section{Acknowledgments}

The authors are extremely grateful to the anonymous referees, whose relevant observations have improved the final version of this paper. This paper was partially supported by grants of the Buenos Aires University (UBA), the National Research Council (CONICET), and the National Research Agency (FONCYT) of Argentina.

\section{References}

[1] B. C. van Fraassen, "A formal approach to the philosophy of science," in Paradigms and Paradoxes: The Philosophical Challenge of the Quantum Domain, R. Colodny, Ed., University of Pittsburgh Press, Pittsburgh, Pa, USA, 1972.

[2] B. C. van Fraassen, "Semantic analysis of quantum logic," in Contemporary Research in the Foundations and Philosophy of Quantum Theory, C. A. Hooker, Ed., Reidel, Dordrecht, The Netherlands, 1973.

[3] B. C. Van Fraassen, "The Einstein-Podolsky-Rosen paradox," Synthese, vol. 29, no. 1-4, pp. 291-309, 1974.

[4] G. Bacciagaluppi and M. Dickson, "Dynamics for modal interpretations," Foundations of Physics, vol. 29, no. 8, pp. 1165-1201, 1999.

[5] O. Lombardi and M. Castagnino, "A modal-Hamiltonian interpretation of quantum mechanics," Studies in History and Philosophy of Modern Physics, vol. 39, no. 2, pp. 380-443, 2008.

[6] M. Castagnino and O. Lombardi, "The role of the Hamiltonian in the interpretation of quantum mechanics," Journal of Physics, vol. 128, Article ID 012014, 2008.

[7] J. S. Ardenghi, M. Castagnino, and O. Lombardi, "Quantum mechanics: Modal interpretation and Galilean transformations," Foundations of Physics, vol. 39, no. 9, pp. 1023-1045, 2009.

[8] O. Lombardi, M. Castagnino, and J. S. Ardenghi, "Quantum mechanics: Interpretation and invariance," Theoria: Revista de Teoria Historia y Fundamentos de la Ciencia, vol. 24, no. 1, pp. 5-28, 2009.

[9] O. Lombardi, M. Castagnino, and J. S. Ardenghi, "The modalHamiltonian interpretation and the Galilean covariance of quantum mechanics," Studies in History and Philosophy of Modern Physics, vol. 41, no. 2, pp. 93-103, 2010.

[10] O. Lombardi, S. Fortin, J. S. Ardenghi, and M. Castagnino, Introduction to the Modal-Hamiltonian Interpretation of Quantum Mechanics, Nova Science, New York, NY, USA, 2010. 
[11] S. Kochen and E. Specker, "The problem of hidden variables in quantum mechanics," Journal of Mathematics and Mechanics, vol. 17, pp. 59-87, 1967.

[12] D. Dieks and P. E. Vermaas, The Modal Interpretation of Quantum Mechanics, Kluwer Academic Publisher, Dordrecht, The Netherlands, 1998.

[13] R. Clifton, "The Properties of Modal Interpretations of Quantum Mechanics," British Journal for the Philosophy of Science, vol. 47, no. 3, pp. 371-398, 1996.

[14] D. Dieks, "Preferred factorizations and consistent property attribution," in Quantum Measurement: Beyond Paradox, R. Healey and G. Hellman, Eds., University of Minnesota Press, Minneapolis, Mo, USA, 1998.

[15] N. L. Harshman and S. Wickramasekara, "Galilean and dynamical invariance of entanglement in particle scattering," Physical Review Letters, vol. 98, no. 8, Article ID 080406, 2007.

[16] N. L. Harshman and S. Wickramasekara, "Tensor product structures, entanglement, and particle scattering," Open Systems and Information Dynamics, vol. 14, no. 3, pp. 341-351, 2007.

[17] P. E. Vermaas, "Unique transition probabilities in the modal interpretation," Studies in History and Philosophy of Modern Physics, vol. 27, no. 2, pp. 133-159, 1996.

[18] A. Elby, "Why "modal" interpretations of quantum mechanics don't solve the measurement problem," Foundations of Physics Letters, vol. 6, pp. 5-19, 1993.

[19] D. Albert and B. Loewer, "Non-ideal measurements," Foundations of Physics Letters, vol. 6, no. 4, pp. 297-305, 1993.

[20] O. Lombardi, "The central role of the Hamiltonian in quantum mechanics: decoherence and interpretation," Manuscrito, vol. 33, no. 1, pp. 307-349, 2010.

[21] O. Lombardi, S. Fortin, M. Castagnino, and J. S. Ardenghi, "Compatibility between environment-induced decoherence and the modal-Hamiltonian interpretation of quantum mechanics," Philosophy of Science. In press.

[22] K. Brading and E. Castellani, "Symmetries and invariances in classical physics," in Philosophy of Physics, J. Butterfield and J. Earman, Eds., North-Holland, Amsterdam, The Netherlands, 2007.

[23] H. Minkowski, "Space and time," in The Principle of Relativity. A Collection of Original Memoirs on the Special and General Theory of Relativity, W. Perrett and G. B. Jeffrey, Eds., Dover, New York, NY, USA, 1923.

[24] H. Weyl, Symmetry, Princeton University Press, Princeton, NJ, USA, 1952.

[25] K. Brading and E. Castellani, Eds., Symmetries in Physics: Philosophical Reflections, Cambridge University Press, Cambridge, UK, 2003.

[26] J. Earman, “Gauge matters," Philosophy of Science, vol. 69, pp. S209-S220, 2002.

[27] J. Earman, "Laws, symmetry, and symmetry breaking: Invariance, conservation principles, and objectivity," Philosophy of Science, vol. 71, no. 5, pp. 1227-1241, 2004.

[28] R. Haag, Local Quantum Physics (Fields, Particles, Algebras), Springer, Berlin, Germany, 1993.

[29] J. M. Lévi-Leblond, "The pedagogical role and epistemological significance of group theory in quantum mechanics," Nuovo Cimento, vol. 4, no. 1, pp. 99-143, 1974.

[30] L. Ballentine, Quantum Mechanics: A Modern Development, World Scientific, Singapore, 1998.

[31] J. S. Ardenghi, M. Castagnino, and R. Campoamor-Stursberg, "The nonrelativistic limit of (central-extended) Poincaŕ group and some consequences for quantum actualization," Journal of Mathematical Physics, vol. 50, no. 10, Article ID 103526, 2009.
[32] J. S. Ardenghi, M. Castagnino, and O. Lombardi, "ModalHamiltonian interpretation of quantum mechanics and Casimir operators: the road toward quantum field theory," International Journal of Theoretical Physics, vol. 50, no. 3, pp. 774-791, 2011.

[33] H. Georgi, Lie Algebras in Particle Physics: From Isospin to Unified Theories, Benjamin-Cummings, Reading, Mass, USA, 1982. 

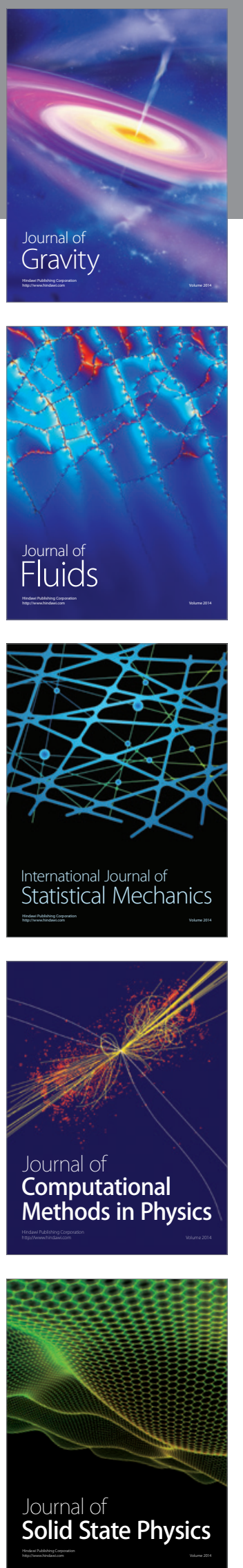

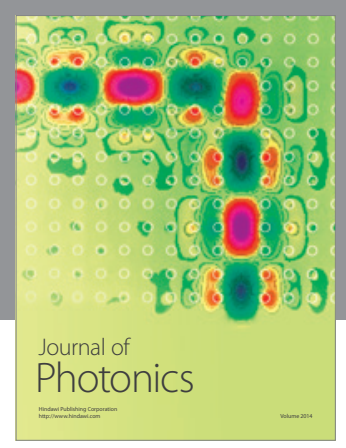

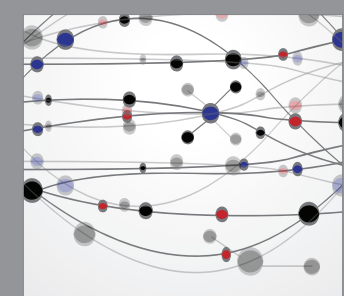

The Scientific World Journal
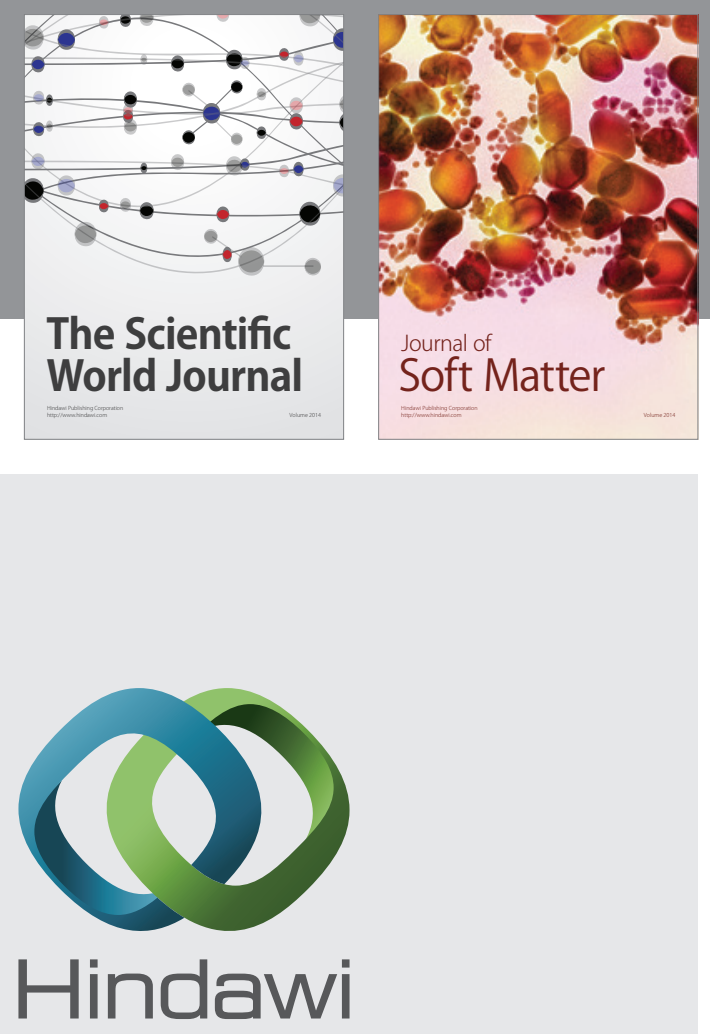

Submit your manuscripts at

http://www.hindawi.com
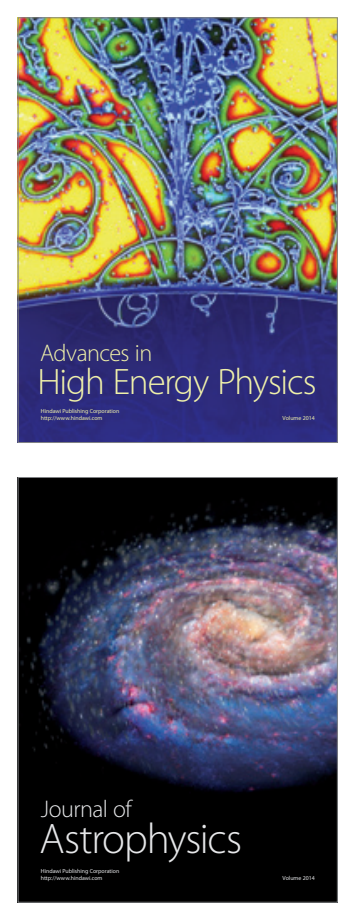
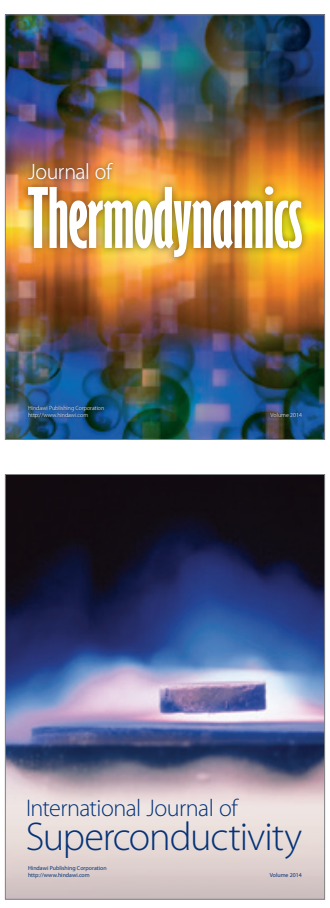
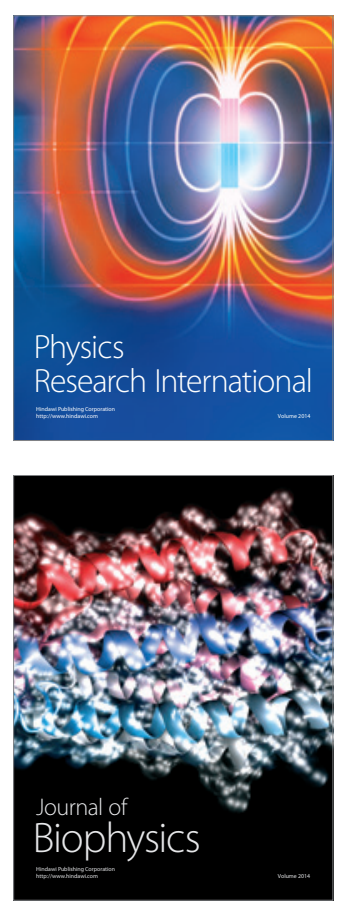
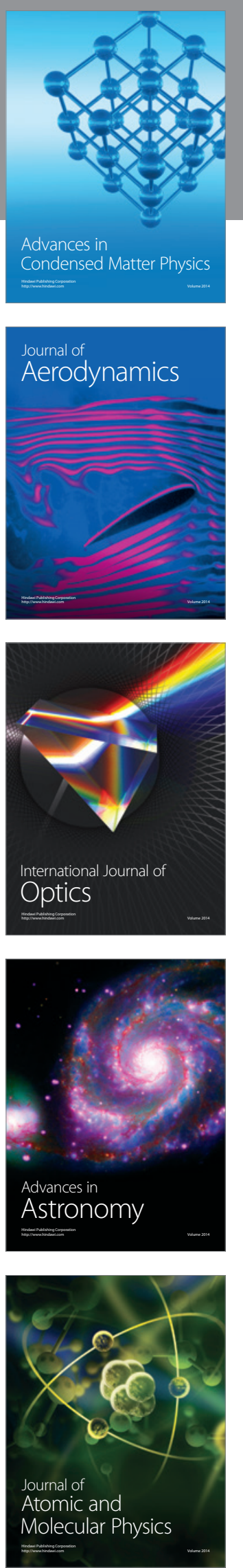